\title{
Comparison of state-of-art phase modulators and parametric mixers in time-lens applications under different repetition rates
}

\author{
Chi Zhang, P. C. Chui, and Kenneth K. Y. Wong* \\ Photonic Systems Research Laboratory, Department of Electrical and Electronic Engineering, \\ The University of Hong Kong, Pokfulam Road, Hong Kong \\ *Corresponding author: kywong@eee.hku.hk
}

Received 24 July 2013; revised 7 October 2013; accepted 15 November 2013; posted 26 November 2013 (Doc. ID 194417); published 17 December 2013

\begin{abstract}
Ever since the discovery of space-time duality, several methods have been developed to perform temporal imaging, and there are two major categories: the quadratic signal onto the phase modulator and the parametric mixer with a linear chirped pump. The features of each mechanism have been thoroughly and quantitatively explored and optimized for certain kinds of applications, but a comparison of some key parameters, especially in the aspect of the repetition rate, is required. In this paper, we will first review the theoretical models and existing performance of these two mechanisms and, consequently, compare them quantitatively in different aspects: the focal group delay dispersion, the pupil size, the effective duty ratio, and the temporal numerical aperture. All these fundamental parameters are related to the repetition rate. The results obtained in this study would provide some important guidelines for the time-lens design, so as to be optimized in different kinds of applications with different repetition rate requirements, such as ultrafast optical communication and real-time bio-imaging systems. (C) 2013 Optical Society of America

OCIS codes: $\quad$ (060.5060) Phase modulation; (110.6915) Time imaging; (190.4410) Nonlinear optics, parametric processes.

http://dx.doi.org/10.1364/AO.52.008817
\end{abstract}

\section{Introduction}

Although the theory of space-time duality and the thorough investigations about the time-lens were not introduced until late 1980s and 1990s [1-5], some time-lens applications have already been demonstrated experimentally before that [ㅎ-요. For example, it was first applied in pulse compression (temporal focusing) in the mid-1960s, with the concept of chirp radar [6,, 7$]$. Generally speaking, space-time duality is based on the mathematical analogy between the paraxial diffraction in the spatial domain and the narrowband dispersion in the temporal domain [1]. The temporal dispersion was

$1559-128 \mathrm{X} / 13 / 368817-10 \$ 15.00 / 0$

(C) 2013 Optical Society of America performed by the diffraction grating pair in the early days [8]. With the more recent development of fiber technology, optical fiber was empowered as an almost ideal candidate for large dispersion $[9,10]$, which greatly accelerated time-lens research. In addition to the temporal dispersion, the linear frequency chirp (sweep) is also required in achieving the time-lens effect. This process is hard to be achieved by a stationary frequency shifter, since it introduces a linear phase sweep, which means constant frequency [11]. Therefore, the linear frequency chirp (sweep) was usually implemented with quadratic phase modulation. Recently there were two major trends in implementing the time-lens. The first one was based on the electro-optic phase modulator: a sinusoidal driving signal was applied. When an optical field aligned with the modulation peak, a locally 
quadratic phase profile was imparted on the field, which enabled it as a time-lens [12]. The other important trend was based on the parametric mixing of frequencies in a nonlinear medium [2,3], where the signal field was mixed with a linear chirped pump to realize the time-lens function. Benefiting from the advance of mode-locked laser, wide bandwidth can be carried out by ultrashort pulses (in the picosecond and femtosecond regimes) and combined with the dispersion management [9]; large quadratic phase profile can be achieved on the chirped pump.

In spatial optics, there are two key parameters to quantify the performance of the space-lens: the focal length and the numerical aperture (NA). The focal length reflects the ability of converging or diverging the diffractive light; here we can identify an analogous parameter in the time-lens counterpart. According to the space-time duality, the focal group delay dispersion (GDD), $\Phi_{f}$, shows the ability of compressing or stretching the dispersed optical field [1]. In regards to the NA, which is similar in the space and time lenses, it quantifies the ability of collecting light and the imaging resolution [4]. In addition to these stationary parameters, since the time-lens is a temporal system, its dynamic features are also of great importance. Here we define a parameter repetition rate, $f_{t}$, which corresponds to the effective operating frame rate of the time-lens. Although it cannot directly affect the time-lens fundamentally, it helps to establish relations and limitations between those fundamental parameters. In this paper, we will mainly focus on the comparison of those fundamental parameters with the repetition rate, over two major time-lens implementation mechanisms.

In early days, the time-lens was first applied in pulse compression (or temporal focusing), without mode-locked cavity [13]. Here time lenses were mainly based on the electro-optic phase modulator $[12,14-16]$, and it achieved several picoseconds pulsewidth pulses output, with a $10 \mathrm{GHz}$ repetition rate. To further enhance the chirped frequency range, higher driving voltage [17] and multipassing the phase modulator [18] were employed to generate sub-picosecond pulses. Later, it was the demonstration of the temporal imaging (packet compression or magnification), which has greatly emphasized the concept of space-time duality. It can still be performed by the phase modulator [12]; however, better performance was achieved by the parametric mixer, which can be performed by the $\chi^{(2)}$ based sum- or difference-frequency generation (SFG, DFG) in some nonlinear crystals $[2, \underline{3}, 19,20]$, and the $\chi^{(3)}$ based fourwave mixing (FWM $)$ in the nonlinear fiber [21] or silicon waveguide [22-25]. Other than temporal imaging, the time-lens system also could be used for Fourier transformation (time-to-frequency conversion), such as the space-lens in the Fourier optics $[26,27]$. These two mechanisms were also employed here for different purposes. For the phase modulator, the modulation bandwidth was usually operated beyond $5 \mathrm{GHz}$ with larger focal GDD [27-29]. While for the parametric mixer, stronger focusing capability (smaller focal GDD) was achieved, but the repetition rate was limited by the mode-locked lasers; regardless, it was implemented by the FWM in a silicon waveguide [30]. For example, a temporal filtering system can be constructed by two cascaded temporal Fourier transformers [31], which can be performed by the phase modulator [32] and the parametric mixer [33]. To give an overview about all these time-lens system based on the two aforementioned parameters, the focal GDD $\Phi_{f}$ and the repetition rate $f_{t}$ (the time-lens effect actually occurs), here we summarize all these references into Fig. 1. All these time lenses are classified into two groups by different chirping mechanisms: the phase modulator (red circle) and the parametric mixer (black diamond). It can be estimated that there is a dashed diagonal line to separate these two groups, except for larger voltage [17] and multipassing phase-modulator cases [18]. Apparently, the phase modulator is favorable to be operated under a higher repetition rate and focal GDD, while the parametric mixer can achieve stronger focusing capability (smaller focal GDD) at lower repetition rate.

Therefore, in this paper, we introduce a parameter repetition rate, as a temporal reference of the timelens system. It helps to derive the quantitative relations among those fundamental parameters and to further identify the intrinsic limitations of the time-lens design. In Section 2, we present a basic review of the mathematical background of the timelens, as well as two implementation mechanisms. In Section 3, we compare these two mechanisms quantitatively in three different aspects: (1) the focal GDD; (2) the pupil size (temporal aperture, the temporal duration of the time-lens effect) and the effective duty ratio (the duty ratio of the temporal aperture over the time period); and (3) the temporal NA. Finally, we summarize the performance of these two sets of time-lens and propose guidelines in the systematic time-lens design. We expect with all these efforts, time-lens will be prosperous in various applications, such as ultrafast signal processing [34],

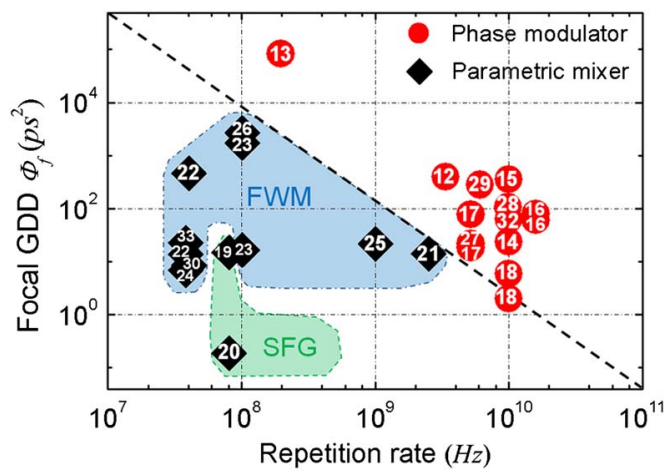

Fig. 1. Relation between the focal GDD $\Phi_{f}$ and the repetition rate $f_{t}$ for the time-lens applications based on electro-optic phase modulator and parametric mixer. The numbers inside each spot refer to the corresponding number in references section. 
frequency domain imaging [35], and temporal cloaking $[\underline{36}, \underline{37]}$.

\section{Principle}

The foundation of the time-lens system is based on the analogy between the paraxial diffraction in spatial domain and narrowband dispersion in the temporal domain [1]. Figure 2 illustrates its basic diagram of a time-lens system, the initial dispersive propagation, followed by a quadratic phase shift, and another dispersive propagation. We will present step-by-step derivations in time and frequency domains, which correspond to the arrows in Fig. 2 . First, we consider the two spools of dispersive fibers, which constitute the input and output of the timelens system. Operated at a lower power level, we can neglect the nonlinear effect within fibers; they will simply introduce a linear dispersion in the frequency domain. $\Phi_{i}$ and $\Phi_{o}$ correspond to the input and the output GDDs (or temporal distance) respectively, which are mathematically analogous to the spreading of a light beam due to the spatial diffraction $[1,38]$. Therefore, if the input signal in time domain is $a(t)[A(\omega)$ in frequency domain], the signal after the first spool of dispersive fiber, $a^{\prime}(t)$, will become

$$
a^{\prime}(t)=\mathfrak{J}^{-1}\left\{A(\omega) G_{i}(\omega)\right\},
$$

where $G_{i}(\omega)=\exp \left(-i \Phi_{i} \omega^{2} / 2\right) \quad$ is the inputdispersion-induced phase shift in the frequency domain. The central time-lens is similar to the space-lens, which produces an instantaneous quadratic phase modulation within the pupil size. As a result, the time-lens can be described by a phase modulation in the form of $-t^{2} / 2 \Phi_{f}$, where the $\Phi_{f}$ is the focal GDD; therefore the time-lens will introduce the quadratic phase shift in the time domain:

$$
t_{f}(t)=\exp \left(-i \frac{t^{2}}{2 \Phi_{f}}\right)
$$

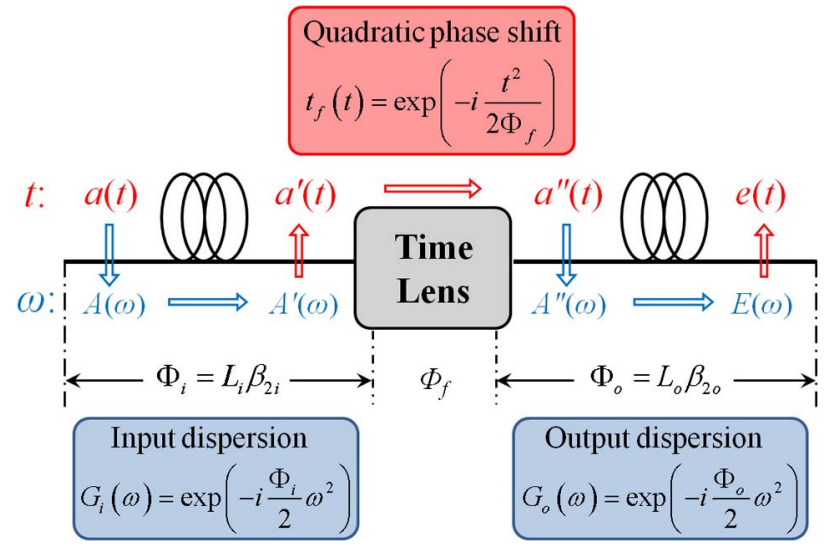

Fig. 2. Schematic diagram of the time-lens. The transformation flow between the time and frequency domain is followed with the arrow directions.
In the frequency domain, it takes the form of $T_{f}(\omega)=\sqrt{-2 \pi i \Phi_{f}} \exp \left(i \Phi_{f} \omega^{2} / 2\right)$. The optical field multiplies with the phase modulation in the time domain and then converts back to the frequency domain. Finally, the optical field passes through the second spool of dispersive fiber and experiences dispersion-induced phase shift $G_{o}(\omega)=\exp \left(-i \Phi_{o} \omega^{2} /\right.$ 2 ) in the frequency domain. Then we substitute the expression of $T_{f}(\omega)$ and $G_{o}(\omega)$ into the calculation, and the output field becomes

$$
\begin{aligned}
e(t)= & \mathfrak{J}^{-1}\left\{\left[A(\omega) G_{i}(\omega) * T_{f}(\omega)\right] G_{o}(\omega)\right\} \\
= & \frac{1}{2 \pi} \int_{-\infty}^{+\infty} \mathrm{d} \omega \exp (i \omega t) G_{o}(\omega) \\
& \cdot \int_{-\infty}^{+\infty} \mathrm{d} \omega^{\prime} A\left(\omega^{\prime}\right) G_{i}\left(\omega^{\prime}\right) T_{f}\left(\omega-\omega^{\prime}\right) \\
= & \int_{-\infty}^{+\infty} \mathrm{d} \omega^{\prime} A\left(\omega^{\prime}\right) G_{i}\left(\omega^{\prime}\right) \exp \left[i \frac{\Phi_{f}}{2} \omega^{\prime 2}\right] \cdot \frac{\sqrt{-2 \pi i \Phi_{f}}}{2 \pi} \\
& \times \int_{-\infty}^{+\infty} \exp \left[i \omega\left(t-\Phi_{f} \omega^{\prime}\right)\right] \exp \left[i \frac{\Phi_{f}-\Phi_{o}}{2} \omega^{2}\right] \mathrm{d} \omega .
\end{aligned}
$$

If $\Phi_{o} \neq \Phi_{f}$, the second integral can be treated as an inverse Fourier transform and can be simplified as

$$
\begin{aligned}
e(t)= & \sqrt{\frac{\Phi_{f}}{\Phi_{f}-\Phi_{o}}} \int_{-\infty}^{+\infty} \mathrm{d} \omega^{\prime} A\left(\omega^{\prime}\right) G_{i}\left(\omega^{\prime}\right) \\
& \times \exp \left[i \frac{\Phi_{f}}{2} \omega^{\prime 2}\right] \exp \left[-\frac{i\left(t-\Phi_{f} \omega^{\prime}\right)^{2}}{2\left(\Phi_{f}-\Phi_{o}\right)}\right] \\
= & \sqrt{\frac{\Phi_{f}}{\Phi_{f}-\Phi_{o}}} \exp \left[\frac{-i t^{2}}{2\left(\Phi_{f}-\Phi_{o}\right)}\right] \\
& \times \int_{-\infty}^{+\infty} \mathrm{d} \omega^{\prime} A\left(\omega^{\prime}\right) \exp \left[\frac{i \Phi_{f} \omega^{\prime}}{\Phi_{f}-\Phi_{o}} t\right] \\
& \times \exp \left[-i\left(\frac{1}{\Phi_{o}}+\frac{1}{\Phi_{i}}-\frac{1}{\Phi_{f}}\right) \frac{\Phi_{i} \Phi_{o} \Phi_{f}}{2\left(\Phi_{f}-\Phi_{o}\right)} \omega^{\prime 2}\right] .
\end{aligned}
$$

Therefore, if it satisfies a similar imaging relation as in the space-lens, $1 / \Phi_{i}+1 / \Phi_{o}=1 / \Phi_{f}$, then the output field can be simplified as

$$
e(t)=2 \pi \sqrt{-\frac{\Phi_{i}}{\Phi_{o}}} \exp \left[i \frac{\Phi_{i}}{2 \Phi_{o} \Phi_{f}} t^{2}\right] a\left(-\frac{\Phi_{i}}{\Phi_{o}} t\right) .
$$

It scales up the input signal in the time axis by a factor of $-\Phi_{o} / \Phi_{i}$. On the other hand, if $\Phi_{i}=\Phi_{o}=\Phi_{f}$, it realizes the function of optical Fourier transformation, which can be illustrated if the output field is expressed in the alternative form: 


$$
\begin{aligned}
e(t)= & \int_{-\infty}^{+\infty} \mathrm{d} \omega^{\prime} A\left(\omega^{\prime}\right) G_{i}\left(\omega^{\prime}\right) \exp \left[i \frac{\Phi_{f}}{2} \omega^{2}\right] \\
& \cdot \frac{\sqrt{-2 \pi i \Phi_{f}}}{2 \pi} \int_{-\infty}^{+\infty} \exp \left[i \omega\left(t-\Phi_{f} \omega^{\prime}\right)\right] \mathrm{d} \omega \\
= & \sqrt{-\frac{2 \pi i}{\Phi_{f}}} A\left(\frac{t}{\Phi_{f}}\right) .
\end{aligned}
$$

Here, the theory of the time-lens system has been introduced briefly. In this paper, we will concentrate on the time-lens part. Two sets of time-lens implementations will be compared in different aspects, including the focal GDD, the temporal NA, the pupil size, and the effective duty ratio. Before these comparisons, we will first introduce the models of each mechanism.

\section{A. Quadratic Phase Modulator Based Time-Lens}

Based on Eq. (2), a quadratic phase modulator could be the reasonable choice for the time-lens $[11,12]$, as illustrated in Fig. 3(a). However, the parabolic electrical signal is approximated by the sinusoidal signal in practical implementation. In other words, only the cusp of the sinusoidal signal can be utilized to approximate the parabolic shape, as shown in the Fig. $3(\mathrm{~b})$. Within this range $\left(\Delta T_{\mathrm{PM}}\right)$, the modulated phase can be expressed by

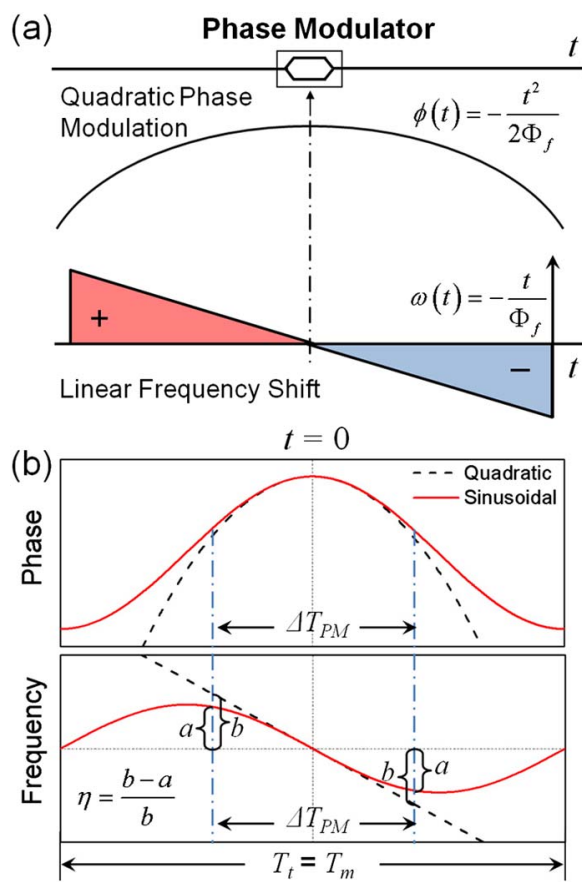

Fig. 3. Principle of the phase modulator is to realize the linear frequency sweep and act as the time-lens: (a) ideal quadratic signal applied to the phase modulator, and linear frequency sweep has been achieved; (b) the cusp of the sinusoidal signal approximates to the quadratic signal, and here the frequency trace is the derivation of phase shift. The error ratio $\eta$ is defined in the frequency domain: the deviation from the real frequency $a$ (solid line) to the ideal frequency $b$ (dashed line), over the ideal frequency $b$, namely $\eta=(b-a) / b$.

$$
\phi(t)=A_{\phi}\left[\cos \left(\omega_{m} t\right)-1\right] \approx-\frac{t^{2}}{2 \Phi_{f}}
$$

where the $A_{\phi}=\pi V / V_{\pi}$ corresponds to the peak of the phase shift, and the $\omega_{m}$ is the modulation frequency of the sinusoidal signal. According to the definition of the focal GDD in Eqs. (2) and (7), we can obtain the relation between the phase shift $\phi(t)$ and the focal GDD $\Phi_{f}$. Therefore, taking the second-order derivative on both sides, the focal GDD $\Phi_{f}$ can be derived as

$$
\Phi_{f \_\mathrm{PM}}=-\frac{1}{\phi^{\prime \prime}(t)} \approx-\frac{1}{\phi^{\prime \prime}(0)}=\frac{1}{A_{\phi} \omega_{m}^{2}} .
$$

Here, the focal GDD $\Phi_{f_{-} \text {PM }}$ is obtained at the cusp of the sinusoidal signal $(t=0)$, and, according to the modulated phase in Eq. (7), $\Phi_{f-P M}$ is determined by the frequency $\omega_{m}$ of the sinusoidal signal and the peak phase shift $A_{\phi}$ introduced by the phase modulator.

\section{B. Parametric Mixer-Based Time-Lens}

An electro-optic phase modulator has been widely applied in time-lens applications, owing to its ease of implementation [11]. However, it is difficult to achieve a large chirped range under low repetition rate (e.g., $f_{t}<5 \mathrm{GHz}$ ); thus recently more and more applications have adopted another parametric mixing procedure, which is based on either $\chi^{(2)}$ or $\chi^{(3)}$ nonlinear processes [2,3]. Newly generated frequency is a mathematical combination of the input frequency and a linear swept pump frequency [38]. In this paper, a parametric mixing procedure called FWM is discussed, especially the degenerate case, where two identical pump photons are annihilated to generate one signal photon and one idler photon $[39,40]$. According to the degenerate FWM relation, $\omega_{i}=$ $2 \omega_{p}-\omega_{s}$, when input signal (frequency, $\omega_{s}$ ) encounters the linear swept pump $\left(\omega_{p}\right)$, a newly generated idler $\left(\omega_{i}\right)$ will contain the conjugated information of the signal $\left(-\omega_{s}\right)$, added with doubled frequency of the swept pump $\left(2 \omega_{p}\right)$, as shown in Fig. $4(\mathrm{~b})$, which is similar to that of the phase modulator.

Now we present the mathematical derivations in details. First, to generate the linear chirped pump, we use a short pulse passing through a spool of dispersive fiber. Before the dispersive fiber, the electric field of the transform-limited Gaussian pulse can be expressed in the form of

$$
\begin{aligned}
a_{p o}(t) & =A_{p o}(t) \exp \left(i \phi_{p o}\right) \\
& =\exp \left[-2 \ln 2\left(\frac{t}{t_{\mathrm{PW}}}\right)^{2}+i \omega_{p o} t\right],
\end{aligned}
$$

where $t_{\mathrm{PW}}$ is the FWHM pulsewidth, and $\omega_{p o}$ is the carrier frequency of the pump. By controlling the peak power to the minimum level, we can then neglect the nonlinear effect along the dispersive fiber and only consider the dispersion-induced phase shift in the frequency domain: $\exp \left[-i \Phi_{p} \omega^{2} / 2\right]$. Here the 
(a)
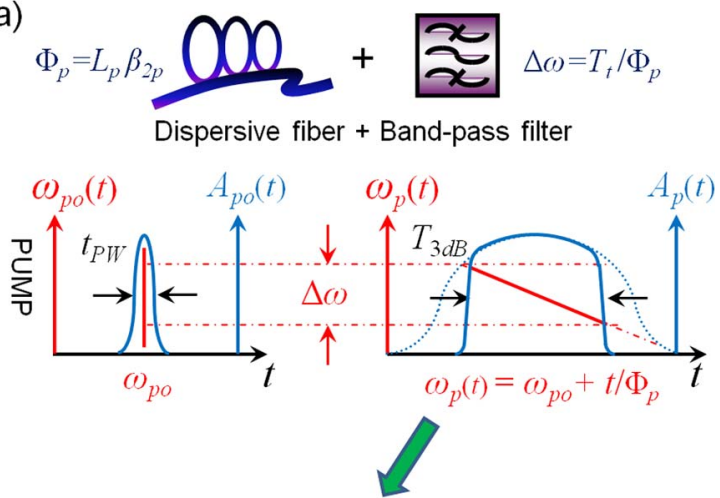

(b)

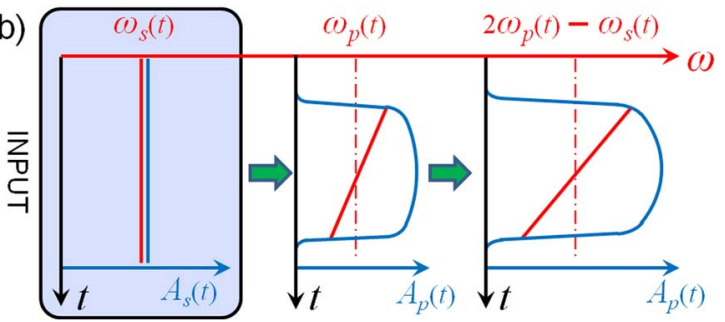

Fig. 4. Principle of the parametric mixer based on FWM to realize the linear frequency sweep and act as the time-lens. (a) Generation of the chirped pump. (b) Frequency transformation during the FWM process.

pump GDD, $\Phi_{p}=L_{p} \beta_{2 p}$, is defined similarly as in the input and output GDDs. $L_{p}$ is the dispersive fiber length, and $\beta_{2 p}$ is the second derivative of the propagation constant at the pump wavelength. Then we multiply the Fourier transform of Eq. (9), with the dispersion component in the frequency domain, and apply the inverse Fourier transform to obtain the stretched pump pulse in the time domain:

$a_{p}(t) \approx \exp \left[-\frac{2 \ln 2}{1+i \varepsilon}\left(\frac{t}{t_{\mathrm{PW}}}\right)^{2}\right] \exp \left[i \frac{\omega_{p o} t}{1+i \varepsilon}\right]=a_{p o}^{\frac{1}{1+i \varepsilon}}(t)$.

It is an approximation because we neglect a constant term $\sqrt{1 /(1+i \varepsilon)}$ in front of the formula, which will simplify the expression without affecting the following derivations; the parameter $\varepsilon$ defined as $\varepsilon=$ $\Phi_{p} / \Phi_{R p}$ is the ratio between the pump GDD $\Phi_{p}$ and the Rayleigh dispersion $\Phi_{R p}=t_{\mathrm{PW}}^{2} /(4 \ln 2)$, which is required to broaden the pump pulsewidth by $\sqrt{2}$ [2]. It is noted that since the initial pump pulsewidth is quite short (picosecond or femtosecond range), the Rayleigh dispersion is small. Also it usually experiences large dispersion-induced phase shift; therefore, we have $\varepsilon \gg 1$. Rearrange the amplitude and phase term of the stretched pump as follows:

$$
\begin{aligned}
a_{p}(t)= & A_{p}(t) \exp \left[i \phi_{p}(t)\right] \\
= & \exp \left[-\left(\frac{t}{t_{\mathrm{PW}}}\right)^{2} \frac{2 \ln 2}{1+\varepsilon^{2}}+\frac{\omega_{p o} t \varepsilon}{1+\varepsilon^{2}}\right] \\
& \times \exp \left[i\left(\frac{t}{t_{\mathrm{PW}}}\right)^{2} \frac{2 \varepsilon \ln 2}{1+\varepsilon^{2}}+i \frac{\omega_{p o} t}{1+\varepsilon^{2}}\right] .
\end{aligned}
$$

The amplitude part describes the temporal shape and position, and it is easy to obtain that the central pump pulse is delayed to $t_{c}=\omega_{p o} \Phi_{p}$, and the FWHM pulsewidth is

$$
T_{3 \mathrm{~dB}}=t_{\mathrm{PW}} \sqrt{1+\varepsilon^{2}} .
$$

The chirped frequency information is the derivative of the phase term in the time domain, and we also shift the central pulse from $t_{c}$ to 0 , namely to replace $t$ by $t+t_{c}$. Also considering $\varepsilon=$ $4 \ln 2 L_{p} \beta_{2 p} / t_{\mathrm{PW}}^{2}$, we can obtain

$$
\begin{aligned}
\omega_{p}(t) & =\frac{\partial \phi_{p}\left(t+t_{c}\right)}{\partial t} \\
& =\frac{1}{1+\varepsilon^{2}}\left[\frac{4 \ln 2 \varepsilon}{t_{\mathrm{PW}}^{2}} t+\frac{4 \ln 2 \varepsilon}{t_{\mathrm{PW}}^{2}} \Phi_{p} \omega_{p o}+\omega_{p o}\right] \\
& =\frac{1}{1+\varepsilon^{2}}\left[\frac{\varepsilon^{2}}{\Phi_{p}} t+\left(1+\varepsilon^{2}\right) \omega_{p o}\right] \\
& =\frac{t}{\left(1+\varepsilon^{-2}\right) \Phi_{p}}+\omega_{p o} .
\end{aligned}
$$

According to the degenerated FWM relation shown in the Fig. $4(\mathrm{~b}), \omega_{i}+\omega_{s}=2 \omega_{p}$, then the frequency of the newly generated idler becomes

$$
\begin{aligned}
\omega_{i}(t) & =2 \omega_{p}(t)-\omega_{s}(t) \\
& =2 \omega_{p o}-\omega_{s}(t)+\frac{2 t}{\Phi_{p}\left(1+\varepsilon^{-2}\right)} .
\end{aligned}
$$

Different from the quadratic phase-modulatorbased time-lens, which only induces the linear chirp, the single-stage FWM-based time-lens also introduces phase conjugation (or spectrum mirror) to the original signal, as the negative sign of the $\omega_{s}(t)$ term shown. It also converts the central wavelength to the idler part. The time-lens-induced swept rate is (the approximation is based on $\varepsilon \gg 1$ )

$$
\omega^{\prime}(t)=\frac{\partial\left[-\omega_{i}(t)-\omega_{s}(t)\right]}{\partial t}=-\frac{2}{\Phi_{p}\left(1+\varepsilon^{-2}\right)} \approx-\frac{2}{\Phi_{p}} .
$$

According to Eq. (2), the relation between the modulated phase and focal GDD is $\phi(t)=-t^{2} / 2 \Phi_{f}$; therefore the second-order derivative can eliminate the time parameter, and the focal GDD can be expressed by the second-order derivative of the phase (or the linear swept rate $\omega^{\prime}(t)=\phi^{\prime \prime}(t)$ ). Similar to the Eq. (ㅇ), as well as the relation in Eq. (15), we can obtain the focal GDD:

$$
\Phi_{f \_W M}=-\frac{1}{\phi^{\prime \prime}(t)}=-\frac{1}{\omega^{\prime}(t)} \approx \frac{\Phi_{p}}{2} .
$$

Noted that the focal GDD achieved by FWM is different from the time-lens based on SFG or DFG, where $\Phi_{f}=\Phi_{p}[\underline{2}, \underline{3}]$. There is a factor of 2 between 
them because the pump frequency is doubly added onto the output in the FWM, while it is the same frequency in the SFG or DFG.

\section{Comparison between Phase Modulator and Parametric Mixer}

The phase modulator and parametric-mixer-based time-lens can introduce the quadratic phase shift (or linear frequency sweep) and realize the function of the time-lens. From Eqs. (8) and (16), we can deduce that the key parameter, focal GDD $\Phi_{f}$, for the time-lens can be adjusted differently. In the case of the phase modulator, a larger driving voltage and higher modulation frequency can help to achieve smaller $\Phi_{f}$ (stronger focusing capability), while for the parametric mixer based on FWM, a smaller pump GDD will result in a smaller $\Phi_{f}$. Moreover, different applications require a different repetition rate (or pupil size) of the time-lens. For example, the bio-imaging is typically in the kilohertz or megahertz range [41] and optical communication system is routinely beyond $10 \mathrm{GHz}$. Therefore, a closer look at the repetition rate is conducted in this section. In particular, we will compare the two aforementioned time-lens mechanisms on different aspects, including the focal GDD, the pupil size, the effective duty ratio, and the temporal NA. All of these will be investigated from the aspect of the repetition rate, and their effects on the systematic design.

\section{A. Focal GDD $\left(\Phi_{f}\right)$ of the Time-Lens}

First, considering the phase-modulator-based timelens, as shown in the Eq. (8), its time period $T_{t}$ should be an integral multiple of the time period of the driving signal $T_{m}=2 \pi / \omega_{m}$. Here we set $T_{t}=T_{m}$ as shown in Fig. 3(b). The phase-modulator-based focal GDD $\Phi_{f_{-} \mathrm{PM}}$ is determined by the modulation frequency $\omega_{m}=2 \pi f_{t}$ and the peak phase shift $A_{\phi}=$ $\pi V / V_{\pi}$ introduced by the phase modulator. In other words, stronger focusing capability (corresponds to shorter focal GDD or faster swept rate) can be achieved with a larger driving voltage. Generally speaking, this voltage cannot exceed a maximum value; suppose the maximum phase shift is $A_{\phi}<A_{\phi \max }=36 \mathrm{rad}$, as well as the relation shown in Eq. (8), we can obtain the following limitation:

$$
\Phi_{f-\mathrm{PM}}=\frac{1}{A_{\phi} \omega_{m}^{2}}>\frac{1}{\left(2 \pi f_{t}\right)^{2} A_{\phi \max }} .
$$

According to current electro-optic devices technology, the bandwidth of the phase modulator could be as high as $100 \mathrm{GHz}$, and here we set $0<f_{t}<40 \mathrm{GHz}$. On the other hand, for the parametric-mixerbased time-lens, the repetition rate is determined by the pump source. To avoid overlapping between stretched neighboring periods, especially when $T_{3 \mathrm{~dB}}>T_{t}$, a filter is required to confine the pulsewidth of the pump within the time period. Under the constant chirped rate as in Eq. (13), the filter bandwidth will become

$$
\Delta \lambda=\frac{\lambda_{0}^{2}}{2 \pi c} \Delta \omega=\frac{\lambda_{0}^{2}}{2 \pi c}\left|\frac{\partial \omega_{p}(t)}{\partial t}\right| T_{t}=\frac{\lambda_{0}^{2}}{4 \pi c f_{t} \Phi_{f-F W M}} .
$$

However, due to the manufacturing process of optical filter, there is a certain constraint on the minimum filter bandwidth, and a conventional filter will usually have $\Delta \lambda>\Delta \lambda_{\min }=0.1 \mathrm{~nm}$. Moreover, as mentioned in the pump-stretching procedure, the approximation based on the pump GDD $\Phi_{p}$ is larger than the Rayleigh dispersion $\Phi_{R p}$; combined with Eq. (16), we will have the repetition rate limitation for the parametric mixer:

$$
\frac{t_{\mathrm{PW}}^{2}}{8 \ln 2}=\frac{\Phi_{R p}}{2}<\Phi_{f_{-} \mathrm{FWM}}<\frac{\lambda_{0}^{2}}{4 \pi c f_{t} \Delta \lambda_{\min }} .
$$

Therefore, when we employ a wider filter bandwidth $\Delta \lambda$, or a larger repetition rate $f_{t}$, this can result in a stronger focusing capability (smaller focal GDD $\left.\Phi_{f \_ \text {FWM }}\right)$, which corresponds to a faster swept rate. According to the relations of the focal GDD $\Phi_{f}$ and the repetition rate $f_{t}$ obtained in Eqs. (17) and (19), we can plot it out in Fig. 5. Generally speaking, the phase modulator works better in a higher repetition rate $f_{t}$ (within the electrical bandwidth), while, for the parametric mixer, it can achieve smaller $\Phi_{f}$ across the whole repetition rate $f_{t}$ range. It is observed that there are four boundaries for the time-lens design as marked in Fig. 5: (1) electrical bandwidth of the phase modulator, which can be improved with the advancement of the electro-optic devices; (2) maximum phase shift introduced by the phase modulator, which can be improved by higher voltage tolerance or multipass design of the phase modulator; (3) minimum filter bandwidth in the parametric mixer, which can be slightly improved by some fiber Bragg gratings with narrower bandwidth; (4) Rayleigh dispersion requirement, which can be largely improved by using ultrashort pump pulses.

Different applications have different requirements in the repetition rate. For example, it is common to

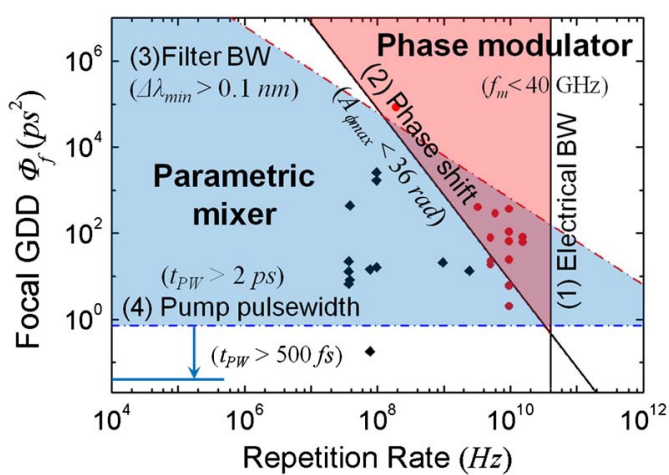

Fig. 5. Effect of repetition rate on the focal GDD range for two time-lens mechanisms. Solid line: constraints on the phase modulator (1) and (2). Dashed-dotted line: constraints on the parametric mixer (3) and (4). Two sets of dots correspond to the relations shown in Fig. 1 . 
use kilohertz or megahertz in bio-imaging applications; within this range, a faster swept rate and wider frequency range can be achieved in a parametric mixer, as shown in Fig. $\underline{5}$, which is essential for those Fourier domain imaging systems [35]. At a higher repetition rate, usually required in optical communication systems, these two kinds of time-lens are joined together, and both of them can be employed in the overlapping area. Other parameters such as the temporal NA and the effective duty ratio will determine the choice of the time-lens mechanism, as illustrated in the following sections.

B. Pupil Size $(\Delta T)$ and the Effective Duty Ratio $\left(R_{t}\right)$ of the Time-Lens

Similar to the space-lens, the time-lens also induces a linear chirped frequency within a certain time range, which is called pupil size. In an ideal case, this range extends across the whole time-lens period $\left(T_{t}=1 / f_{t}\right)$, while it is not the case in the implementation. Here we define another parameter, pupil size $(\Delta T)$, which corresponds to the frequency sweep range under a certain error-ratio threshold. In the case of the phase-modulator-based time-lens, only the cusp of the sinusoidal signal can be approximated to the ideal parabolic pulse. It introduces significant error chirp beyond this time range (pupil size), which can be denoted as $\Delta T_{\mathrm{PM}}$, while the error tolerance is $\eta$ at the two edges $\left(t= \pm \Delta T_{\mathrm{PM}} / 2\right)$, as shown in Fig. $3(\mathrm{~b})$. As a result, we obtain the chirped frequency from the derivative of the phase shift as follows:

$$
\omega(t)=-\frac{t}{\Phi_{f_{-} \mathrm{PM}}}(1-\eta)=-A_{\phi} \omega_{m} \sin \left(\omega_{m} t\right) .
$$

Therefore, the pupil size can be expressed as

$$
\Delta T_{\mathrm{PM}}=\frac{2 \pi \operatorname{sinc}^{-1}(1-\eta)}{\omega_{m}},
$$

where $\sin ^{-1}$ is the inverse of the normalized sinc function. It can be noted that $T_{m}$ is the time period of the modulation signal, $T_{m}=2 \pi / \omega_{m}$, and the effective duty ratio can be expressed as $R_{t_{-} \mathrm{PM}}=\Delta T_{\mathrm{PM}} / T_{m}=\operatorname{sinc}^{-1}(1-\eta)$. Its pupil size is limited in the red shaded region at the bottom of Fig. 6(a), and it is the same case for the effective duty ratio, as shown in Fig. 6(b). It is noticed that here we employed the sinusoidal waveform in the phase-modulator-based time-lens, owing to its ease in achieving a higher repetition rate. As a comparison, the time-lens driven by the Gaussian waveform was analyzed under the same error tolerance, and generally the sinusoidal waveform achieves better duty ratio. The fitted result is shown as the dashed blue line in Fig. 6(b) [42]. Therefore it is common to insert a Mach-Zehnder modulator in front of the phase-modulator-based time-lens to achieve a precise linear chirp [14], especially when confining the working range as a pupil and enhancing the timelens accuracy [1-3]. Furthermore, some tailored
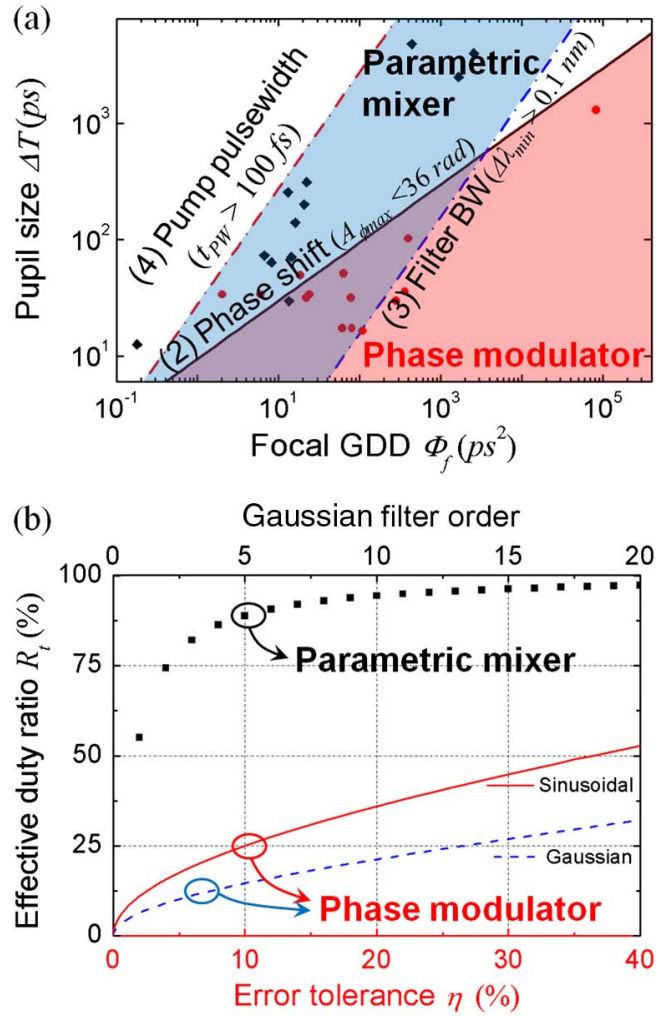

Fig. 6. (a) Pupil size versus the focal GDD: constraints on the phase modulator (red-shaded area enclosed by solid line), and constraints on the parametric mixer (blue-shaded area enclosed by dashed-dotted lines). Two sets of dots correspond to the relations shown in Fig. 1. (b) The effective duty ratio of two time-lens mechanisms, phase modulator (red solid: sinusoidal, blue dash: Gaussian); parametric mixer (dots).

waveforms can help to achieve much better accuracy and higher effective duty ratio $[43,44]$.

In the case of the parametric-mixer-based timelens, to avoid any overlapping between neighboring periods, a filter is applied to confine the stretched pump pulsewidth. The pupil size is also limited by the minimum filter bandwidth and the initial pump pulsewidth. The pupil size of these two mechanisms varies as a function of the focal GDD, as shown in Fig. 6(a). Based on the previous discussion, the parametric mixer is usually operated with a smaller focal GDD. The parametric mixer (shaded area enclosed by the dashed-dotted lines) can definitely achieve larger pupil size, which also can be comfortably controlled by the filter bandwidth. However, the practical optical filter cannot be the perfect squared shape. In other words, this time-lens cannot achieve the full duty ratio. For instance, the filter shape can be described by a Gaussian function with a different order $(n)$. To minimize the influence from neighboring pulses, we set the power at least $10 \mathrm{~dB}$ below the central peak power at the edge of each time-lens period. The effective duty ratio can be expressed as

$$
R_{t \_ \text {FWM }}<(\log 2)^{\frac{1}{2 n}}
$$


Here the maximum effective duty ratio of the parametric-mixer-based time-lens is determined by the sharpness of the filter, which acts as a pupil [3]. The comparison of the two mechanisms is shown in Fig. 6(b). It is noticeably easier to achieve a larger effective duty ratio by the parametric-mixer-based time-lens, which also has a flexible duty ratio tuning range. For example, a fifth-order Gaussian filter is enough to achieve $90 \%$ of $R_{t}$ by parametric mixer, while one has to suffer $10 \%$ error tolerance to achieve only $25 \%$ of $R_{t}$ in the case of phase modulator.

\section{Temporal Numerical Aperture $\left(\mathrm{NA}_{t}\right)$ of the Time-Lens}

In spatial optics, the NA characterizes the range of angles over which the system can accept or emit light, while in the time-lens, the $\mathrm{NA}_{t}$ can be defined as the allowable range of chirped frequencies. NA is commonly used in microscopy to indicate the resolving power of a lens and its light-collecting ability. When the lens is focused at infinity, the spatial NA can be approximated by $\mathrm{NA}_{\mathrm{s}} \approx D / 2 f$. In the case of time-lens, the diameter of pupil $D$ corresponds to the temporal pupil size $\Delta T$. Substituting the focal length $f$ by the focal GDD $\Phi_{f}$, we can then obtain the $\mathrm{NA}_{t}=\Delta T / 2 \Phi_{f}$; namely the chirped frequency range. The ideal resolution, $\delta t$, of the time-lens system is shown to be inversely proportional to the temporal NA, i.e., $\delta t=4 \ln 2 / \mathrm{NA}_{t}$, without considering the misfocus and any aberrations [3,4]. A lens with a larger $\mathrm{NA}_{t}$ will be able to resolve finer details and collect more light and benefit the applications such as Fourier domain imaging [35], but also limit the detection depth range, according to the features of the confocal imaging system [41].

Here we explore how these two systems perform in the aspect of temporal NA due to their own constraints. In the case of phase modulator, the driving signal has limited electrical bandwidth $\left(f_{t \max }=40 \mathrm{GHz}\right)$; therefore the temporal NA (or chirped frequency range) is restricted by

$$
\mathrm{NA}_{t \_\mathrm{PM}}=\frac{\Delta T_{\mathrm{PM}}}{2 \Phi_{f}}<2 \pi^{2} A_{\phi} \operatorname{sinc}^{-1}(1-\eta) f_{t \max } .
$$

Here we set $A_{\phi}=5 \mathrm{rad}, \eta=10 \%$, and $f_{t}=$ $10 \mathrm{GHz}$; then the widest chirped frequency range that can be achieved is $2.465 \times 10^{11} \mathrm{rad} / \mathrm{s}$ (around $0.314 \mathrm{~nm}$ ), which is insufficient for most wideband applications. The limitations of the phase shift and the electrical bandwidth restrict the $\mathrm{NA}_{t}$ of the phase modulator within the triangle area in Fig. 7 (solid line). Furthermore, the ideal resolution can be simply described by

$$
\delta t_{\mathrm{PM}}=\frac{\ln 2}{\pi^{2} A_{\phi} \operatorname{sinc}^{-1}(1-\eta) f_{t}},
$$

which is inversely proportional to the repetition rate $f_{t}$; namely a wider electrical bandwidth helps us to achieve finer temporal resolution. Consider $f_{t}=$ $40 \mathrm{GHz}$ and $A_{\phi}=36 \mathrm{rad}$, the phase-modulator-based resolution is $\delta t_{\mathrm{PM}}=196 \mathrm{fs}$. In the case of a parametric-mixer-based time-lens, if there is no overlap between the neighboring pulses (without filtering), the $\mathrm{NA}_{t}$ can be expressed as

$$
\mathrm{NA}_{t-\mathrm{FWM}}=\frac{T_{3 \mathrm{~dB}}}{2 \Phi_{f}}=\frac{4 \ln 2}{t_{\mathrm{PW}} \sqrt{1+\varepsilon^{-2}}} \approx \frac{4 \ln 2}{t_{\mathrm{PW}}} .
$$

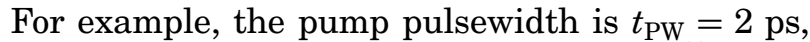
then the temporal NA can be $1.39 \times 10^{12} \mathrm{rad} / \mathrm{s}$ (chirped frequency range around $1.77 \mathrm{~nm}$ ). It is larger than the phase-modulator-induced chirpedfrequency range, and the temporal resolution is equal to the original pump pulsewidth $t_{\mathrm{PW}}$. However, in a higher repetition rate or faster swept rate, pump pulse easily interferes with the adjacent pulse, and a filter with bandwidth $\Delta \lambda$ is required to confine it within the time period. The temporal NA will inevitably decrease and degrade the resolution. The dashed-dotted lines in Fig. 7 confine the NA area of the parametric mixer, which is limited by the pump pulsewidth and the filter bandwidth, and it is noticed that a larger temporal NA could be achieved by even a shorter pump pulsewidth. Since the filter bandwidth in the frequency domain is $\Delta \omega_{f}=2 \pi c \Delta \lambda / \lambda_{0}^{2}$, the filtered $\mathrm{NA}_{t-\mathrm{FWM}}$ and the degraded resolution will become

$$
\delta t_{\mathrm{FWM}}=\frac{4 \ln 2}{\Delta \omega_{f}}=\frac{2 \ln 2 \lambda_{0}^{2}}{\pi c \Delta \lambda} .
$$

Considering the filter bandwidth $\Delta \lambda=1 \mathrm{~nm}$ at $1550 \mathrm{~nm}$, the resolution of a parametric mixer is $\delta t_{\mathrm{FWM}}=3.54 \mathrm{ps}$. Therefore, constructing a highfidelity parametric mixing time-lens system requires efficient conversion of all input and output spectral components to their sum or difference frequencies. The conversion efficiency of FWM can be greatly enhanced if the phase-matching condition is satisfied, which usually requires that the pump wavelength is close to the zero-dispersion wavelength of the highly nonlinear dispersion-shifted fiber $\left(\mathrm{GVD}_{p} \approx 0\right)$

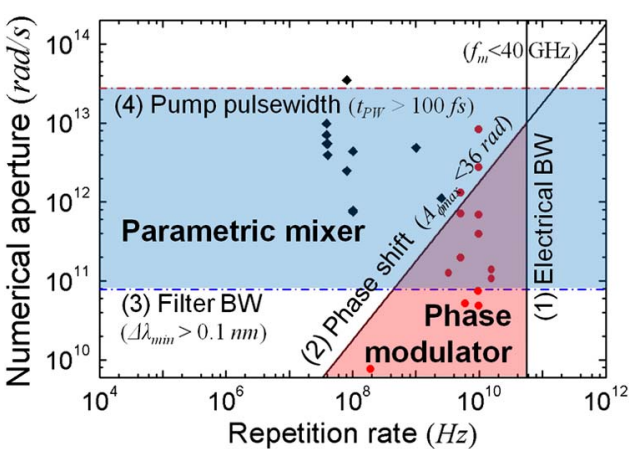

Fig. 7. Effect of repetition rate on the temporal NA performance for two time-lens mechanisms. Solid line: constraints on the phase modulator (1) and (2). Dashed-dotted line: constraints on the parametric mixer (3) and (4). Two sets of dots correspond to the relations shown in Fig. 1 . 
[39]. If a wide wavelength range is involved in this procedure, some dispersion-flattened highly nonlinear fiber is required to ensure the phase-matching condition is satisfied over the whole wavelength range [39].

\section{Discussion}

Recent developments in time-lens technology has demonstrated the space-time duality in many applications, including pulse compression, temporal imaging, Fourier transformation, and temporal filtering. An electro-optic phase modulator and parametric mixer with a linear-chirped pump were employed for the time-lens applications, and the theory and implementations of both mechanisms have been well developed separately. Generally speaking, these two time-lens mechanisms have an obvious difference in their implementation. First, the phase modulator is easy to implement, while the parametric mixer requires a whole stage of sweep-source-pumped FWM; second, the focal GDD is easy to be adjusted in the phase modulator through the driven voltage, while in the parametric mixer, it is impossible to continuously change the dispersion. Last, after the phase modulator, the central wavelength is preserved, while, after a parametric mix, the output field is usually in a different wavelength. The wavelengthpreserving operation requires multistage configurations [26]. Therefore the phase modulator excels in the implementation aspect, without considering the specification of some fundamental parameters.

In this paper, these two time-lens mechanisms are compared with some fundamental parameters, including the focal GDD $\Phi_{f}$, the temporal NA, the pupil size $\Delta T$, and the effective duty ratio $R_{t}$, associated with the repetition rate $f_{t}$. The performance of all these parameters in the two mechanisms are summarized in Fig. 8 , and the shaded area is the preferred operation region: the phase-shift value determines the boundary of the phase modulator, while the filter sharpness and the initial pump pulsewidth determine the parametric mixer operation range. First, the parametric mixer is easy for achieving a

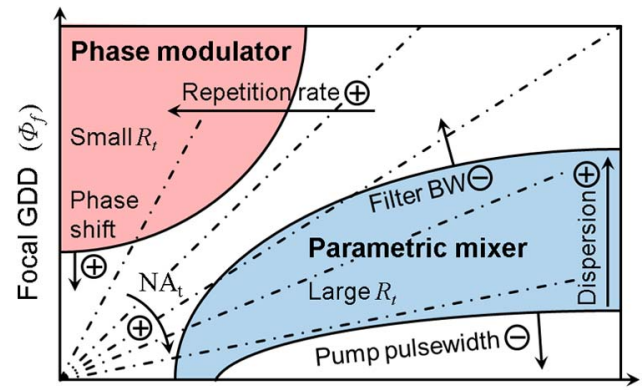

Pupil size $(\Delta T)$

Fig. 8. Design guidelines of two time-lens mechanisms. The red-shaded area on the top-left corner corresponds to the phase modulator, and the blue-shaded area on the bottom-right corner corresponds to the parametric mixer. The dashed-dotted lines refer to the $\mathrm{NA}_{t}$ value, and the direction of arrows reflects the increase/decrease of the corresponding parameters. stronger focusing capability (smaller focal GDD $\Phi_{f}$ ) and is controllable with pump dispersion, though it is still confined by the initial pump pulsewidth and the minimum filter bandwidth. On the contrary, the phase modulator results in a larger focal GDD $\Phi_{f}$, and stronger focusing capability can be achieved by higher driving voltage or multipassing the phase modulator $[17,18]$. Second, the parametric mixer has a much larger temporal NA, as well as better resolution, than the phase modulator configuration. It is clearly shown in Fig. 8 that the slope of the dasheddotted line corresponds to the value of $\mathrm{NA}_{t}$. This result explains why the parametric mixer mechanism achieved sharper imaging features in the previous temporal imaging demonstrations, though the filter used to avoid overlapping under a higher repetition rate will degrade its performance. While for the phase modulator, a larger $\mathrm{NA}_{t}$ and improved resolution are achieved under a larger phase shift, such as increasing the driving voltage applied at the phase modulator. However, a large $\mathrm{NA}_{t}$ also makes the parametric mixer easier to misfocus, and the adjustment of the dispersive fiber length is more difficult than increasing the voltage applied to the phase modulator. On the other hand, the sinusoidal phase modulator introduces order-of-magnitude-higher aberrations than the higher-order dispersion in the parametric mixer [4]. Third, the sinusoidalapproximated driving signal of the phase modulator limited the effective duty ratio $R_{t}$ to be smaller than that of the parametric mixer, which can be flexibly controlled through the filter bandwidth. In addition, since the parametric mixer is usually operated under a lower repetition rate, a larger pupil size is guaranteed. It provides important guidelines for the time-lens implementation, which is essential in designing high-level time-lens applications in a systematic approach.

The work described in this paper was partially supported by grants from the Research Grants Council of the Hong Kong Special Administrative Region, China (project HKU 717212E).

\section{References}

1. B. H. Kolner, "Space-time duality and the theory of temporal imaging," IEEE J. Quantum Electron. 30, 1951-1963 (1994).

2. C. V. Bennett and B. H. Kolner, "Principles of parametric temporal imaging-part i: system configurations," IEEE J. Quantum Electron. 36, 430-437 (2000).

3. C. V. Bennett and B. H. Kolner, "Principles of parametric temporal imaging-part ii: system performance," IEEE J. Quantum Electron. 36, 649-655 (2000).

4. C. V. Bennett and B. H. Kolner, "Aberrations in temporal imaging," IEEE J. Quantum Electron. 37, 20-32 (2001).

5. B. H. Kolner and M. Nazarathy, "Temporal imaging with a time lens," Opt. Lett. 14, 630-632 (1989).

6. J. R. Klauder, A. C. Price, S. Darlington, and W. J. Albersheim, "The theory and design of chirp radars," Bell Syst. Tech. J. 39, 745-808 (1960).

7. W. J. Caputi, "Stretch: a time-transformation technique," IEEE Trans. Aerosp. Electron. Syst. AES-7, 269-278 (1971)

8. E. B. Treacy, "Optical pulse compression with diffraction gratings," IEEE J. Quantum Electron. 5, 454-458 (1969).

9. T. Jannson and J. Jannson, "Temporal self-imaging effect in single-mode fibers," J. Opt. Soc. Am. 71, 1373-1376 (1981). 
10. T. Jannson, "Real-time Fourier transformation in dispersive optical fibers," Opt. Lett. 8, 232-234 (1983).

11. C. F. Buhrer, D. Baird, and E. M. Conwell, "Optical frequency shifting by electro-optic effect," Appl. Phys. Lett. 1, 46-49 (1962).

12. B. H. Kolner, "Active pulse compression using an integrated electro-optic phase modulator," Appl. Phys. Lett. 52, 1122-1124 (1988).

13. J. E. Bjorkholm, E. H. Turner, and D. B. Pearson, "Conversion of $\mathrm{cw}$ light into a train of subnanosecond pulses using frequency modulation and the dispersion of a near resonant atomic vapor," Appl. Phys. Lett. 26, 564-566 (1975).

14. J. van Howe, J. Hansryd, and $\mathrm{C}$. Xu, "Multiwavelength pulse generator using time-lens compression," Opt. Lett. 29, 1470-1472 (2004).

15. M. Romagnoli, P. Franco, R. Corsini, A. Schiffini, and M. Midrio, "Time-domain Fourier optics for polarization-mode dispersion compensation," Opt. Lett. 24, 1197-1199 (1999).

16. J. Azana, N. K. Berger, B. Levit, and B. Fischer, "Reconfigurable generation of high-repetition-rate optical pulse sequences based on time-domain phase-only filtering," Opt. Lett. 30, 3228-3230 (2005).

17. A. A. Godil, B. A. Auld, and D. M. Bloom, "Picosecond timelenses," IEEE J. Quantum Electron. 30, 827-837 (1994).

18. J. van Howe, J. H. Lee, and C. Xu, "Generation of $3.5 \mathrm{~nJ}$ femtosecond pulses from a continuous-wave laser without mode locking," Opt. Lett. 32, 1408-1410 (2007).

19. C. V. Bennett, R. P. Scott, and B. H. Kolner, "Temporal magnification and reversal of $100 \mathrm{~Gb} / \mathrm{s}$ optical data with an up-conversion time microscope," Appl. Phys. Lett. 65, 2513-2515 (1994).

20. C. V. Bennett and B. H. Kolner, "Upconversion time microscope demonstrating $103 \times$ magnification of femtosecond waveforms," Opt. Lett. 24, 783-785 (1999).

21. P. J. Almeida, P. Petropoulos, B. C. Thomsen, M. Ibsen, and D. J. Richardson, "All-optical packet compression based on time-to-wavelength conversion," IEEE Photon. Technol. Lett. 16, 1688-1690 (2004).

22. M. A. Foster, R. Salem, Y. Okawachi, A. C. Turner-Foster, M. Lipson, and A. L. Gaeta, "Ultrafast waveform compression using a time-domain telescope," Nat. Photonics 3, 581-585 (2009).

23. Y. Okawachi, R. Salem, M. A. Foster, A. C. Turner-Foster, M. Lipson, and A. L. Gaeta, "High-resolution spectroscopy using a frequency magnifier," Opt. Express 17, 5691-5697 (2009).

24. R. Salem, M. A. Foster, A. C. Turner-Foster, D. F. Geraghty, M. Lipson, and A. L. Gaeta, "High-speed optical sampling using a silicon-chip temporal magnifier," Opt. Express 17, 4324-4329 (2009).

25. R. Salem, M. A. Foster, A. C. Turner, D. F. Geraghty, M. Lipson, and A. L. Gaeta, "Optical time lens based on fourwave mixing on a silicon chip," Opt. Lett. 33, 1047-1049 (2008).

26. C. Zhang, J. Xu, P. C. Chui, and K. K. Y. Wong, "Parametric spectro-temporal analyzer (PASTA) for real-time optical spectrum observation,” Sci. Rep. 3, 2064 (2013).
27. M. T. Kauffman, W. C. Banyai, A. A. Godil, and D. M. Bloom, "Time-to-frequency converter for measuring picosecond optical pulses," Appl. Phys. Lett. 64, 270-272 (1994).

28. J. Azana, N. K. Berger, B. Levit, and B. Fischer, "Spectrotemporal imaging of optical pulses with a single time lens," IEEE Photon. Technol. Lett. 16, 882-884 (2004).

29. N. K. Berger, B. Levit, S. Atkins, and B. Fischer, "Timelens-based spectral analysis of optical pulses by electro-optic phase modulation," Electron. Lett. 36, 1644-1646 (2000)

30. M. A. Foster, R. Salem, D. F. Geraghty, A. C. Turner, M. Lipson, and A. L. Gaeta, "Silicon-chip-based ultrafast optical oscilloscope," Nature 456, 81-84 (2008).

31. A. W. Lohmann and D. Mendlovic, "Temporal filtering with time lenses," Appl. Opt. 31, 6212-6219 (1992).

32. T. Sakano, K. Uchiyama, I. Shake, T. Morioka, and K. Hagimoto, "Large-dispersion-tolerance optical signal transmission system based on temporal imaging," Opt. Lett. 27, $583-585$ (2002).

33. O. Kuzucu, Y. Okawachi, R. Salem, M. A. Foster, A. C. Turner-Foster, M. Lipson, and A. L. Gaeta, "Spectral phase conjugation via temporal imaging," Opt. Express 17, 20605-20614 (2009).

34. J. van Howe and $\mathrm{C}$. $\mathrm{Xu}$, "Ultrafast optical signal processing based upon space time dualities," J. Lightwave Technol. 24, 2649-2662 (2006).

35. S. H. Yun, G. J. Tearney, J. F. de Boer, N. Iftimia, and B. E. Bouma, "High-speed optical frequency-domain imaging," Opt. Express 11, 2953-2963 (2003).

36. M. Fridman, A. Farsi, Y. Okawachi, and A. L. Gaeta, "Demonstration of temporal cloaking," Nature 481, 62-65 (2012).

37. J. M. Lukens, D. E. Leaird, and A. M. Weiner, "A temporal cloak at telecommunication data rate," Nature $\mathbf{4 9 8}$, 205-208 (2013).

38. G. P. Agrawal, Nonlinear Fiber Optics, 4th ed. (Academic, 2007).

39. M. E. Marhic, Fiber Optical Parametric Amplifiers, Oscillators and Related Devices (Cambridge University, 2007).

40. C. Zhang, K. K. Y. Cheung, P. C. Chui, K. K. Tsia, and K. K. Y. Wong, "Fiber-optical parametric amplifier with high-speed swept pump," IEEE Photon. Technol. Lett. 23, 1022-1024 (2011).

41. J. B. Pawley, Handbook of Biological Confocal Microscopy, 3rd ed. (Springer Berlin, 2006).

42. B. H. Kolner, "Electro-optic time lenses for shaping and imaging optical waveforms," in Modulators for Optical Communications, Science, Technology, and Applications, A. Chen and E. Murphy, eds. (CRC Press, 2012), pp. 427-454.

43. R. Wu, V. R. Supradeepa, C. M. Long, D. E. Leaird, and A. M. Weiner, "Generation of very flat optical frequency combs from continuous-wave lasers using cascaded intensity and phase modulators driven by tailored radio frequency waveforms," Opt. Lett. 35, 3234-3236 (2010).

44. L. E. Munioz-Camuniez, V. Torres-Company, J. Lancis, J. Ojeda-Castaneda, and P. Andres, "Electro-optic time lens with an extended time aperture," J. Opt. Soc. Am. B 27, 2110-2115 (2010). 